\title{
Core Binding Factor Acute Myeloid Leukemia
}

National Cancer Institute

\section{Source}

National Cancer Institute. Core Binding Factor Acute Myeloid Leukemia. NCI Thesaurus. Code $C 122688$.

Acute myeloid leukemia characterized by the presence of $\mathrm{t}(8 ; 21)(\mathrm{q} 22 ; \mathrm{q} 22)$ or inv(16) $(p 13 q 22) / t(16 ; 16)(p 13 ; q 22)$. These cytogenetic abnormalities result in disruption of the transcription factor CBF, which is a regulator of normal hematopoiesis. 